may be all that is open to us in such organic structural problems : there may be nothing essentially novel in its present explicit statement. Yet even Pasteur was an amateur.

The phenomena which used to be grouped under the name chemotaxy, and also the mode of transfer of hormones, might arise from cognate activities.

$$
\begin{aligned}
& \text { Holywood, } \\
& \text { Co. Down. } \\
& \text { Oct. } 31 \text {. } \\
& { }^{1} \text { H. Ramage, NATURE, 138, } 762 \text { (Oct. 31, 1936). }
\end{aligned}
$$

\section{Gaseous Combustion}

IT was shown by Mallard and Le Chatelier more than fifty years ago that, during the passage of flame through an inflammable gaseous mixture, two types of flame movement were clearly distinguishable, namely, the initial uniform slow movement and the very rapid movement known as detonation which is set up-if the mixture is strong enough-after the flame has travelled some distance.

Prof. W. A. Bone states that there is now much evidence for the view that, during the initial slow movement, combustion is far from being complete in the flame front, but that as the detonation stage is reached, combustion in the flame front becomes practically complete ${ }^{1}$. With this view I am in agreement, provided that incomplete combustion is not identified with incomplete combination but is to be accounted for by a stable form of latent energy formed in the flame front and left behind in the flame gases. The nature of the latent energy must for the present be left vague, but it seems probable that it will be found to be located in metastable molecules of some kind or other which result from the reactions in the flame front.

The reason why incomplete combination would appear to be ruled out is that it must remain constant in amount for a very long time after the flame front has passed if it is to explain flame temperature ${ }^{2}$ and explosion and heat-loss ${ }^{3}$ measurements, and this, of course, is most unlikely. Furthermore, had the photographic studies by H. B. Dixon, Bone and others of flame-travel in tubes been made with tubes of very large diameter, so that the cooling of the inflamed gases did not take place rapidly, it would have been found that the duration of the after-glow of the inflamed gases was so long as to be inconsistent with an explanation in terms of incomplete combination. Indeed, in some explosion experiments made in a very large vessel, the after-glow continued for at least 14 seconds 4 , and it is a fair inference that had the explosion vessel been still larger so that the cooling would have taken place much more slowly, the luminosity would have lasted for a longer time than this.

It has been shown that the latent energy left behind in the flame gases decreases markedly with the pressure at which the gases are burnt ${ }^{5}$ and, since the pressure in the flame front during detonation is very high, it is to be expected that the latent energy in the detonation phase would be much less than in the initial slow movement phase. Assuming also, as would appear to be legitimate, that the latent energy decreases with distance of travel of the flame front, a completely satisfactory explanation is obtained as to why there is a smaller proportion of latent energy in large vessel explosions than in flames ${ }^{3}$.

Our experiments, so far as they have gone, have shown latent energies varying between 1 per cent and 10 per cent of the heat of combustion in explosions ${ }^{3}$, and between 6 per cent and 28 per cent of the heat of combustion in flames ${ }^{5}$. Doubtless further experiments will show that these limits may be extended in either direction. There seems little doubt that the amount of the heat of combustion remaining in flame gases as latent energy depends not only on the pressure at which combustion takes place in the flame front, but also upon the nature of the combustible gases and the diluent gases ${ }^{3,5}$.

Ordinary gas calorimetry shows that the latent energy, which our experiments show to be long-lived, entirely disappears during the passage of the flame gases through the calorimeter. The after-glow shows that it tends to disappear when the temperature is sufficiently high to produce collisions of the kind which will enable the metastable molecules to attain the ground state, but this is clearly a very slow process, and it seems probable that the bulk of it disappears when the flame gases come into contact with surface. Preliminary experiments designed to test this have been apparently successful, but they have not yet reached a stage at which publication is desirable. Meanwhile, attention may be directed to Dr. F. W. Lanchester's calorimeter experiment, in which there was no surface and in which he could only account for at most 85 per cent of the heat of combustion $^{6}$. It is of interest, too, in this connexion to point out that flame gases, after having been cooled to a temperature so low as $200^{\circ} \mathrm{C}$. in the cylinder of a gas engine, have been made luminous again by adiabatic compression ${ }^{4}$. The adiabatic compression ignition experiment of Tizard and Pye in which explosion took place on the second compression? would also appear to be relevant.

Engineering Department,

$$
\text { W. T. David. }
$$
University,

Leeds.

Nov. 9.

1 Phil. Trans. Roy. Soc., 230, 364.

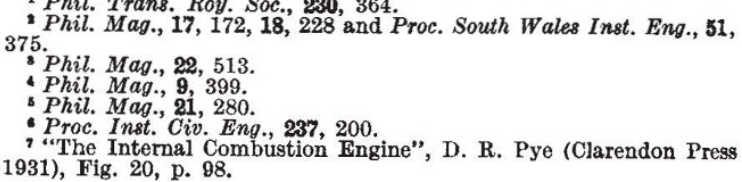

\section{Red and Sunlit Auroras and the State of the Upper Atmosphere}

ON October 16 this year, a strong auroral display was observed at Oslo. It commenced as an arc fairly low on the northern sky, but at intervals it took the form of a drapery-shaped band with pronounced ray structure, and sometimes-for example, between $22^{\mathrm{h}}$ and $23^{\mathrm{h}}$ in the evening-rays of quite unusual length (several hundred kilometres) were observed.

These observations show that extremely long rays may appear also on the night side of the earth, and that they are not restricted to regions exposed directly to sunlight.

Our direct measurements from auroral band spectra gave a night temperature of $-30^{\circ}$ to $-47^{\circ} \mathrm{C}$. in the auroral region. Thus the slow rate of decrease of density from $100 \mathrm{~km}$. and upwards as shown by the long auroral rays cannot be accounted for by a high temperature, but as I have stated in previous papers, it must be due to an electrical state of the atmosphere, resulting from the action of ultra-violet light or corpuscular rays entering the atmosphere during auroral displays and magnetic disturbances. 